\title{
Importance of prognostic nutritional index in on-pump coronary artery bypass graft surgery
}

\section{Arif Gücü®}

Department of Cardiovascular Surgery, University of Health Sciences, Bursa Yüksek İhtisas Training and Research Hospital, Bursa, Turkey

\section{ABSTRACT}

Objectives: A simple and appropriate risk index is still required to show the patient's nutritional status undergoing coronary artery bypass graft $(\mathrm{CABG})$ surgery. This study aimed to evaluate the Prognostic Nutritional Index (PNI) value as a predictor of in-hospital mortality in patients undergoing CABG surgery.

Methods: In this study, we scanned the medical data of 742 patients' who underwent on-pump CABG surgery retrospectively. Patients' were divided into two groups based on the PNI cut-off value (high-risk group, PNI < $45.85, \mathrm{n}=230$; low-risk group, $\mathrm{PNI} \geq 45.85, \mathrm{n}=512$ ).

Results: To analyze the factors affecting in-hospital mortality in the postoperative period, univariate and multivariate logistic regression analysis was performed. In univariate analysis, advanced age (Odds ratio (OR): $1.219,95 \%$ confidence interval (CI): $1.194-2.669, p<0.001$ ), left ventricular ejection fraction (LVEF) (OR: 3.471, 95\% CI: 2.854-6.927, $p<0.001$ ), total perfusion time (OR: 0.876, 95\% CI: 0.690-0.954, $p=0.012$ ), intra-aortic balloon pump (IABP) use (OR: 2.148, 95\% CI: 1.394-2.889, p =0.002), preoperative high creatinine (OR: 1.229, 95\% CI: 1.066-2.118, $p=0.019$ ), low lymphocyte count (OR: $0.879,95 \%$ CI: 0.789 $0.945, p=0.017$ ), low albumin (OR: $1.682,95 \% \mathrm{CI}: 1.433-2.765, p=0.003)$, high C-reactive protein (CRP) (OR: $1.0 .790,95 \%$ CI: $0.678-0.927, p=0.042$ ) and low PNI (OR: 1.290, 95\% CI: $1.119-1.654, p<0.001$ ) were correlated with the postoperative mortality. In multivariate logistic regression analysis, advanced age (OR: $1.145,95 \%$ CI: 1.110-1.938, $p=0.017$ ), LVEF (OR: 2.916, 95\% CI: 1.768-4.928, $p<0.001$ ), IABP use (OR: $1.880,95 \% \mathrm{CI}: 1.350-2.554, p=0.032$ ) and PNI (OR: $0.932,95 \% \mathrm{CI}: 0.889-0.978, p=0.004)$ were independent predictors of mortality.

Conclusions: In on-pump CABG surgery, postoperative mortality is associated with low preoperative PNI, and can be a useful and suitable parameter for preoperative risk evaluation.

Keywords: Prognostic nutritional index, nutritional status, CABG surgery, cardiopulmonary bypass, risk factors

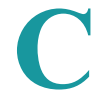
oronary artery disease (CAD) is lead to death and disability throughout the world [1]. Coronary artery bypass graft (CABG) surgery is one of the most important treatment methods of CAD. Although new developments in the treatment of CAD have reduced mortality rates, the perioperative and hospital mortality and morbidity rates of patients remain high [2-4]. In CABG surgery, the mortality and mortality rates of patients are affected by many factors $[5,6]$. A simple and appropriate risk index is still required to show the 
patient's nutritional status undergoing CABG surgery. Clinical nutritional status is an indirect indicator of patient resistance. This relationship has been demonstrated in patients undergoing gastrointestinal system and malignancy surgery [7, 8]. Various methods such as Mini Nutrition Assessment, Malnutrition Universal Screening Tool and Subjective Global Assessment have been developed to determine the nutritional status of patients undergoing malignancy surgery. The Prognostic Nutritional Index (PNI) is the most widely used method for determining nutritional status, and low PNI has been shown as a predictor of postoperative mortality and morbidity in malignant diseases $[9,10]$. The PNI, which was simplified by Onodera et al. [11], was calculated based on the serum albumin concentration and lymphocyte count. Some comprehensive studies evaluate the effects of preoperative nutritional factors on coronary artery surgery outcomes and vascular diseases [12, 13]. However, the importance of PNI in CABG surgery is not yet clear.

This study aims to determine of the value of PNI as a predictor of in-hospital mortality and morbidity in patients undergoing on-pump CABG surgery.

\section{METHODS}

A total of 742 patients were included in this retrospective study that underwent on-pump CABG surgery at our institute between January 2016 and January 2020. In order to avoid the possible effects of the COVID-19 pandemic, the study was terminated in January 2020, when no pandemic patients were detected in our country yet. The research was approved by the institutional ethics committee of the Bursa Yuksek Ihtisas Training and Research Hospital. The study was conducted in accordance with the Declaration of Helsinki Ethical Principles and Good Clinical Practices.

Demographic characteristic and clinical outcomes of the patients were retrospectively collected by searching our hospital database. Other data, body mass index (BMI), cross-clamp (X-clamp) time, cardiopulmonary bypass (CPB) time, preoperative left ventricular ejection fraction (LVEF), intra-aortic balloon pump (IABP) usage, intensive care unit (ICU) stays, postoperative hospital-acquired infection and stroke were noted. We defined six types of infections after cardiac surgery as hospital-acquired infections based on O'Keefe et al. [14]. These included urinary tract infections, pneumonia, harvest site infections, superficial sternal wound infections, deep sternal wound infections, and sepsis. We also noted that stroke events after surgery.

\section{Blood parameter analysis}

Peripheral blood samples taken on the first day of hospitalization for all patients were noted. The complete blood cell count analysis was performed using automatic blood analyzer (Beckmann Coulter LH 780) and biochemical analyzes were performed using automatic biochemical analyzer (Cobas 6000, Manheim).

\section{Calculation of PNI}

The preoperative nutritional status of the patients was assessed using the PNI classification. PNI was calculated according to the formula: $10 \times$ serum albu$\min +0.005 \times$ total lymphocyte count [13]. To determine the optimal cut-off value for PNI, a method described by Budczies et al. [15] was used.

\section{Surgical Technique}

All operations were performed under general anaesthesia with a median sternotomy. Standard CPB was used in mild hypothermia $\left(32^{\circ} \mathrm{C}\right)$ with aorta-venous two-stage cannulation. Cardioplegic arrest was achieved. Cardiopulmonary bypass was provided by roller pumps at a flow rate of $2-2.4 \mathrm{~L} / \mathrm{min} / \mathrm{m}^{2}$ and a membrane oxygenator (Maquet, Getinge Group, Rastatt, Germany). Arterial filters were used in all operations. All distal anastomoses were performed under aortic cross-clamp (ACC), while proximal anastomoses were performed using the partial clamp technique. All patients received warm potassium-free blood cardioplegia before the ACC was removed. All patients were transferred to the cardiovascular surgery intensive care unit after the operation.

\section{Statistical Analysis}

Continuous data were expressed as the mean \pm standard deviation. Categorical data were expressed as the number and percentage. Continuous variables were analyzed with Student's t-test (for normally distributed data) or Mann-Whitney U test (for non-nor- 
mally distributed data). The difference in categorical variables was tested using the chi-squared test. Univariate and multivariate logistic regression analysis were performed to determine potential risk factors for postoperative mortality. The predictive value of PNI for mortality was determined with Receiver Operating Characteristic (ROC) curve analysis and Area Under Curve (AUC) was calculated. All statistical analyses were performed using the SPSS package for Windows version 21 (SPSS Inc., Chicago, IL, USA). A $p$ value of $<0.05$ was accepted statistically significant.

\section{RESULTS}

The overall study consisted of 742 patients. Inhospital mortality occurred in $43(5.4 \%)$ patients. The mean and median levels of the PNI were 49.92-7.44 and 49.81 (45.58-54.27). The cut-off point was set at 45.85. When the cut-off point was 45.85 , the specificity was $58 \%$, the sensitivity was $70.7 \%$ satisfactory, and patients were divided into two groups according to this cut-off point (high-risk group; PNI $<45.85$, n $=230$; low-risk group; PNI $\geq 45.85, \mathrm{n}=512$ ).
The low-risk group and the high-risk group patients were compared, and there was a significant difference in age (low- risk group: $66 \pm 0.75$ vs. highrisk group: $62.62 \pm 0.43, p=0.001)$. The BMI was similar between the groups $(26.97 \pm 0.19$ vs. $26.51 \pm$ $0.13 ; p=0.179)$. Preoperative LVEF was statistically lower in the high-risk group $(49.67 \pm 0.62$ vs. $51.20 \pm$ $0.43 ; p=0.018$ ). There was no difference between two groups in terms of hypertension, DM and COPD rates. Comparisons of the groups regarding the laboratory data revealed that the high-risk group had significantly lower hemoglobin and albumin levels $(p=0.001)$. Preoperative serum creatinine levels were differences between groups and the high-risk group had significantly higher creatinine levels $(1.25 \pm 0.08$ vs. $0.94 \pm 0.02$; $p=0.001)$. Comparison of demographic features and basic blood values of patients in groups are demonstrated in Table 1.

Operative X-clamp time ( $p=0.661), \mathrm{CPB}$ time $(p$ $=0.143)$ was similar between the groups, but IABP usage $(p=0.001)$, and ICU stay $(p=0.011)$ significantly higher in the high-risk group. Since the infection was broadly categorized in our study, we compared hospital-acquired infection between groups.

Table 1. Comparison of demographic features and basic blood values of patients in groups

\begin{tabular}{|c|c|c|c|}
\hline Features & High-risk group & Low-risk group & $p$ values \\
\hline Male, n (\%) & $185(80.5)$ & $409(79.9)$ & 0.527 \\
\hline Age (year) & $66 \pm 0.75$ & $62.62 \pm 0.43$ & 0.001 \\
\hline Preoperative LVEF (\%) & $49.67 \pm 0.62$ & $51.20 \pm 0.43$ & 0.018 \\
\hline $\mathrm{BMI}\left(\mathrm{m}^{2} / \mathrm{kg}\right)$ & $26.97 \pm 0.19$ & $26.51 \pm 0.13$ & 0.179 \\
\hline Hypertension, n (\%) & $132(57.3)$ & $277(54.1)$ & 0.108 \\
\hline Diabetes mellitus, $\mathrm{n}(\%)$ & $48(20.8)$ & $93(18.1)$ & 0.227 \\
\hline COPD, n (\%) & $42(18.2)$ & $79(15.4)$ & 0.090 \\
\hline Hemoglobin (gr/dL) & $12.15 \pm 0.14$ & $13.66 \pm 0.08$ & 0.001 \\
\hline $\operatorname{WBC}\left(\times 10^{3}\right)$ & $9.36 \pm 0.26$ & $9.22 \pm 0.11$ & 0.431 \\
\hline $\mathrm{Neu}\left(\mathrm{x} 10^{3}\right)$ & $7.11 \pm 0.22$ & $6.05 \pm 0.07$ & 0.001 \\
\hline $\operatorname{Lym}\left(\times 10^{3}\right)$ & $1.42 \pm 0.04$ & $2.32 \pm 0.04$ & 0.001 \\
\hline Platelets $\left(\times 10^{3}\right)$ & $240.24 \pm 0.54$ & $247.30 \pm 2.96$ & 0.011 \\
\hline $\mathrm{CRP}(\mathrm{mg} / \mathrm{L})$ & $22.17 \pm 2.19$ & $9.31 \pm 0.71$ & 0.001 \\
\hline $\operatorname{Cre}(\mathrm{mg} / \mathrm{dL})$ & $1.25 \pm 0.08$ & $0.94 \pm 0.02$ & 0.001 \\
\hline Total protein $(\mathrm{g} / \mathrm{dl})$ & $6.47 \pm 0.04$ & $7.07 \pm 0.03$ & 0.001 \\
\hline Albumin (g/dL) & $3.39 \pm 0.03$ & $4.04 \pm 0.02$ & 0.001 \\
\hline
\end{tabular}

Data are shown as mean \pm standard deviation or $\mathrm{n}(\%) . \mathrm{WBC}=$ White blood cell, $\mathrm{CRP}=\mathrm{C}$-reactive protein, Cre $=\mathrm{Creatinine}$, $\mathrm{Neu}=$ Neutrophil, Lym $=$ Lymphocyte, $\mathrm{LVEF}=$ Left ventricular ejection fraction, BMI = Body Mass Index 
Table 2. Operative and postoperative features of patients

\begin{tabular}{lccc}
\hline Characteristic & High-risk group & Low-risk group & $\boldsymbol{p}$ values \\
\hline X-clamp time (min) & $66.41 \pm 1.58$ & $64.32 \pm 0.9$ & 0.661 \\
CPB time (min) & $95.20 \pm 2.05$ & $90.54 \pm 1.22$ & 0.143 \\
IABP usage, n (\%) & $29(12.6 \%)$ & $24(4.6)$ & $\mathbf{0 . 0 0 1}$ \\
ICU stay (days) & $3.71 \pm 0.16$ & $3.09 \pm 0.13$ & $\mathbf{0 . 0 1 1}$ \\
Mortality, $\mathrm{n}(\%)$ & $25(10.8)$ & $18(3.5)$ & $<\mathbf{0 . 0 0 1}$ \\
Morbidity & & & \\
Hospital-acquired infection, $\mathrm{n}(\%)$ & $59(25.6)$ & $62(12.1)$ & $\mathbf{0 . 0 0 1}$ \\
Stroke, $\mathrm{n}(\%)$ & $26(3.7)$ & $36(7)$ & 0.052 \\
\hline
\end{tabular}

Data are shown as mean \pm standard deviation or $\mathrm{n}(\%)$. X- clamp $=$ Cross clamp, $\mathrm{CPB}=$ Cardiopulmonary bypass, IABP $=$ Intra-aortic balloon pump, ICU = Intensive care unit

There was a significantly higher tendency of hospitalacquired infection in the high-risk group $(59 ; 25.6 \%$ vs. $62 ; 12.1 \% ; p=0.001)$. Postoperative stroke was similar between the groups $(26 ; 11.3 \%$ vs. $36 ; 7 \% ; p$ $=0.052)$ (Table 2).

To analyze the factors affecting in-hospital mortality in the postoperative period, univariate and multivariate logistic regression analysis was performed (Table 3). In univariate analysis, advanced age (OR [odds ratio]: $1.219,95 \%$ CI [confidence interval]: 1.194-2.669, $p<0.001$ ), LVEF (OR: 3.471, 95\% CI: 2.854-6.927, $p<0.001$ ), total perfusion time (OR:
0.876, 95\% CI: 0.690-0.954, $p=0.012$ ), IABP use (OR: 2.148, 95\% CI: 1.394-2.889, p=0.002), preoperative high creatinine (OR: 1.229, 95\% CI: 1.066$2.118, p=0.019)$, low lymphocyte count (OR: 0.879, 95\% CI: 0.789-0.945, $p=0.017$ ), low albumin (OR: $1.682,95 \%$ CI: $1.433-2.765, p=0.003)$, high CRP (OR: $0.790,95 \% \mathrm{CI}: 0.678-0.927, p=0.042$ ) and low PNI (OR: 1.290, 95\% CI: 1.119-1.654, $p<0.001$ ) were correlated with the postoperative mortality. In multivariate logistic regression analysis, advanced age (OR: $1.145,95 \%$ CI: $1.110-1.938, p=0.017)$, LVEF (OR: $2.916,95 \%$ CI: 1.768-4.928, $p<0.001$ ), IABP

Table 3. Logistic regression analysis to identify factors affecting postoperative mortality

\begin{tabular}{lcccccc}
\hline \multirow{2}{*}{ Variables } & \multicolumn{3}{c}{ Univariate analysis } & \multicolumn{3}{c}{ Multivariate analysis } \\
& $\boldsymbol{p}$ value & $\begin{array}{c}\text { Exp (B) } \\
\text { Odds Ratio }\end{array}$ & $\begin{array}{c}\mathbf{9 5 \%} \text { CI } \\
\text { Lower-Upper }\end{array}$ & $\boldsymbol{p}$ value & $\begin{array}{c}\text { Exp (B) } \\
\text { Odds Ratio }\end{array}$ & $\begin{array}{c}\text { 95\% CI } \\
\text { Lower-Upper }\end{array}$ \\
\hline Age & $<\mathbf{0 . 0 0 1}$ & 1.219 & $1.194-2.669$ & $\mathbf{0 . 0 1 7}$ & 1.145 & $1.110-1.938$ \\
Hypertension & 0.221 & 1.317 & $0.975-1.786$ & -- & -- & -- \\
\hline COPD & 0.190 & 0.895 & $0.667-1.110$ & -- & -- & -- \\
LVEF & $<\mathbf{0 . 0 0 1}$ & 3.471 & $2.854-6.927$ & $<\mathbf{0 . 0 0 1}$ & 2.916 & $1.768-4.928$ \\
\hline Total perfusion time & $\mathbf{0 . 0 1 2}$ & 0.876 & $0.690-0.954$ & 0.345 & 0.790 & $0.690-1.134$ \\
IABP use & $\mathbf{0 . 0 0 2}$ & 2.148 & $1.394-2.889$ & 0.032 & 1.880 & $1.350-2.554$ \\
\hline Creatinin & $\mathbf{0 . 0 1 9}$ & 1.229 & $1.066-2.118$ & 0.314 & 1.110 & $0.890-1.440$ \\
Lymphocyte count & $\mathbf{0 . 0 1 7}$ & 0.879 & $0.789-0.945$ & -- & -- & -- \\
\hline Albumin & $\mathbf{0 . 0 0 3}$ & 1.682 & $1.433-2.765$ & -- & -- & -- \\
CRP & $\mathbf{0 . 0 4 2}$ & 0.790 & $0.678-0.927$ & 0.410 & 0.914 & $0.776-1.190$ \\
\hline PNI & $<\mathbf{0 . 0 0 1}$ & 1.290 & $1.119-1.654$ & $\mathbf{0 . 0 0 4}$ & 0.932 & $0.889-0.978$ \\
\hline
\end{tabular}

COPD $=$ Chronic obstructive pulmonary disease, $\mathrm{LVEF}=$ Left ventricular ejection fraction, IABP $=$ Intraaortic balloon pump, $\mathrm{CRP}=\mathrm{C}$ reactive protein, $\mathrm{PNI}=$ Prognostic nutritional index 


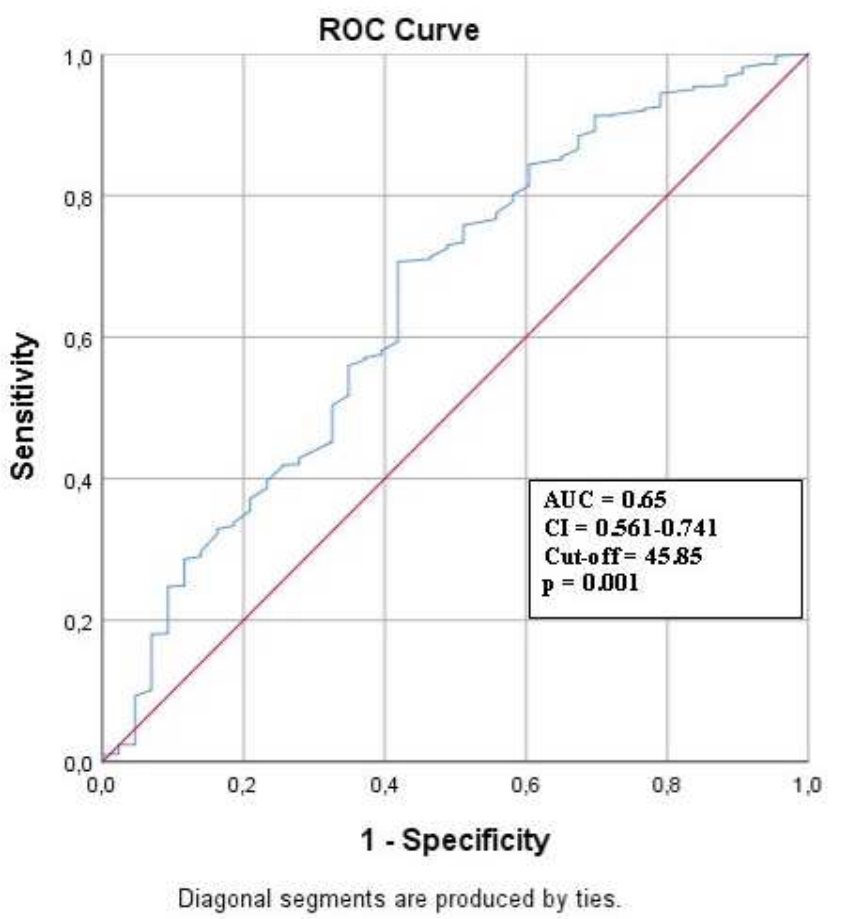

Fig. 1. Data figure of the area under the curve (AUC), confidence interval (CI), and cut-off values in receiver-operating characteristic (ROC) curve analysis for prognostic nutritional index.

use (OR: $1.880,95 \% \mathrm{CI}: 1.350-2.554, p=0.032)$ and PNI (OR: 0.932, 95\% CI: 0.889-0.978, $p=0.004$ ) were independent predictors of mortality.

In the receiver-operating characteristic analysis, PNI lower than 45.85 predicted in-hospital mortality with $70.7 \%$ sensitivity and $58 \%$ specificity [area under the curve: $0.651,95 \%$ confidence interval: 0.561 $0.741 ; p=0.001$ ] (Fig. 1). In-hospital outcomes were significantly worse in low-risk group (Fig. 2).

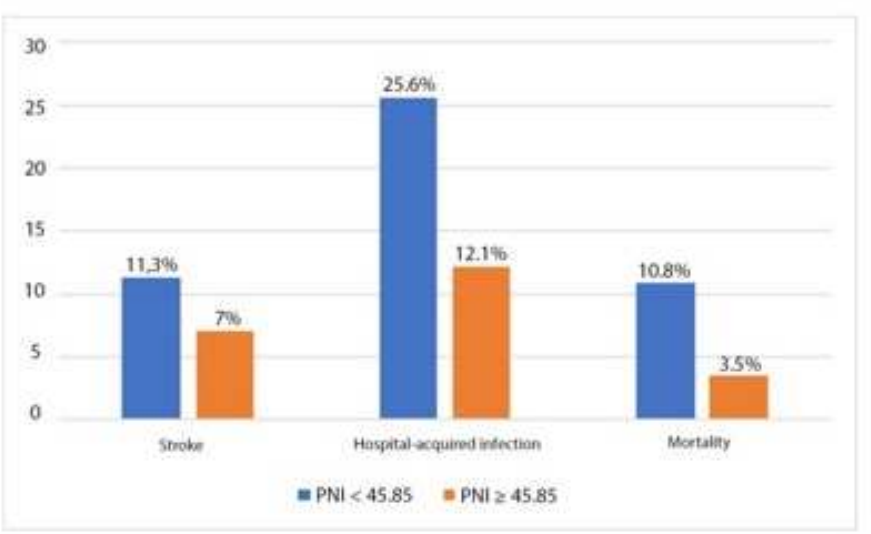

Fig. 2. In-hospital outcomes were significantly worse when the PNI was lower.

\section{DISCUSSION}

CABG surgery is one of the most important treatment methods of CAD. Operation success is related to individual risk factors as well as technical skills. In this current study, we showed that PNI value, which is an important indicator of malnutrition status, is an independent predictor of postoperative mortality in addition to known risk factors such as age and low LVEF value.

Patients with poor nutritional status before cardiac surgery have been higher postoperative morbidity and mortality rates [16]. In the literature some studies have demonstrated the importance of energy and protein metabolism in the early period after heart surgery and documented significant post-operative consumption of macronutrients and micronutrients $[16,18]$. Sufficient nutritional therapy has been proposed to improve patient outcomes by maintaining energy metabolism and promoting improved wound healing after surgery [19]. Lomivorotov et al. found that depending on the used nutrition screening tool, the percentage of malnourished cardiac surgery patients before surgery ranged between 4.6-19.1\% [18]. The present findings point to high malnutrition rates in cardiac patients, and it is very important to consider the nutritional profiles of these patients before surgery and simultaneously, and to draw more attention to the concept of individual diet for pre-operative optimization in these patients [20, 22]. Therefore, preoperative evaluation of nutritional status in patients at high risk of developing postoperative complications may guide to consider postoperative nutritional interventions. Studies evaluating the relationship of BMI, albumin, and prealbumin levels have demonstrated that they are independent predictors of mortality and morbidity after coronary artery and valve surgery [23, 24].

The PNI, calculated based on the serum albumin concentration and lymphocyte count of peripheral blood was designed by Buzby et al. [7] in 1980 and simplified by Onodera et al. [11] in 1984. Lymphocytes are significant part of the immune system, and the prognostic role of lymphocyte count has been previously investigated in cardiovascular diseases [25, 27]. Lymphopenia is an important mortality predictor in patients undergoing CABG surgery [28]. Albumin is a serum protein that is a good indicator of a patient's nutritional status, and it makes up the majority of the 
serum total protein and is mainly responsible for the serum osmotic pressure. Also albumin has antioxidant and anti-inflammatory properties in scavenging reactive oxygen radicals and limiting their production [29] In particular, postoperative hypoalbuminemia is an independent risk factor for postoperative outcome in patients undergoing CABG surgery [30].

PNI was originally designed to determine immunonutrition status and has been widely used to assess surgical risk, particularly in patients with malignancy and gastrointestinal operations $[7,8,31]$. Several studies have reported that lower PNI levels are significantly associated with higher mortality in patients with cardiovascular disease [32]. PNI can be used to predict patients' outcomes before cardiac surgery and to choose an appropriate surgical strategy. Given all these data, the management of patients with low PNI may require a "Cardio-metabolic Team" approach to optimized patient care prior to surgery. In the cardio-metabolic team consisting of cardiologist, internal medicine, dietician, cardiovascular surgeon and needed additional branches. It is clear that pre-operative metabolic optimal support is particularly important in this low- and middle-income patient. On the other hand, in patients with low PNI, cardiac surgery plan can be re-evaluated by the cardio-metabolic team. Less invasive surgery may be appropriate if possible. There are various studies in the literature investigating the effect of PNI value on clinical outcomes after cardiac operations. In a study conducted by Lee et al. [33], the prognostic role of PNI value in predicting early clinical outcomes after cardiac surgery was investigated. In this study, the authors divided the patients into two groups according to the value they determined as cut-off (46.13). At the end of the study, early mortality rates were found to be higher in the group with low PNI values. In addition, the mean length of stay on mechanical ventilation and length of stay in the intensive care unit were found to be significantly higher in this group [33]. In our study patients' were seperated into two groups based on the PNI cutoff value (high-risk group, PNI $<45.85$; low-risk group, PNI $\geq 45.85$ ). There was a significantly higher tendency of hospital-acquired infection in the highrisk group $(59 ; 25.6 \%$ vs. $62 ; 12.1 \% ; p=0.001)$ and postoperative stroke was similar between the groups $(26 ; 11.3 \%$ vs. $36 ; 7 \% ; p=0.052)$. In multivariate analysis PNI value was found as an independent pre- dictor of postoperative mortality.

An increasing number of elderly people are accepted for elective CABG surgery. When low preoperative PNI is detected in elderly patients undergoing elective coronary artery surgery, postoperative methods such as serum albumin supplementation, dietary maintenance or nutritional support should be considered to improve the nutritional status of patients [34].

Our research demonstrated that a low PNI value affects postoperative mortality and morbidity. There was significantly different a higher tendency for postoperative hospital-acquired infection in the high-risk group. PNI may be used to predict patients' outcomes before coronary artery surgery and select an adequate surgical strategy. In the case of patients with a low PNI, less invasive surgery may be suitable if possible.

\section{Limitations}

Our study does not include the effects of preoperative nutritional support on postoperative mortality and morbidity in patients with low PNI values. The fact that this is a single-center study with a retrospective design is the most important limitation. Further studies are needed to show the results of preoperative nutritional support in elective cases.

\section{CONCLUSION}

Preoperative low PNI level was statistically significantly associated with postoperative mortality and morbidity in cardiac surgery. According to the results we obtained in our study, we firmly believe that PNI is a useful and suitable parameter for preoperative assessment of nutritional status and should be regarded in managing the indication and strategy in on-pump CABG surgery.

\section{Authors' Contribution}

Study Conception: AG; Study Design: AG; Supervision: AG; Funding: AG; Materials: AG; Data Collection and/or Processing: AG; Statistical Analysis and/or Data Interpretation: AG; Literature Review: AG; Manuscript Preparation: AG and Critical Review: AG.

\section{Conflict of interest}

The author disclosed no conflict of interest during 
the preparation or publication of this manuscript.

\section{Financing}

The author disclosed that they did not receive any grant during conduction or writing of this study.

\section{Acknowledgments}

I would like to thank the Bursa Yuksek Ihtisas Research and Training Hospital cardiovascular surgery team for all these operations.

\section{REFERENCES}

1. Yalcin M, Ay D, Turk T, Yavuz S, Ozyazicioglu AF. Impact of previous percutaneous coronary intervention on postoperative outcomes of coronary artery bypass grafting. Eur Res J 2016;2:170-6.

2. Hannan EL, Racz MJ, Walford G, Jones RH, Ryan TJ, Bennett $\mathrm{E}$, et al. Long-term outcomes of coronary-artery bypass grafting versus stent implantation. N Engl J Med. 2005;352:2174-83.

3. Engin M, Aydin C. Investigation of the Effect of HATCH score and coronary artery disease complexity on atrial fibrillation after on-pump coronary artery bypass graft surgery. Med Princ Pract 2021;30:45-51.

4. Weymann A, Ali-Hasan-Al-Saegh S, Popov AF, Sabashnikov A, Mirhosseini SJ, Liu T, et al. Haematological indices as predictors of atrial fibrillation following isolated coronary artery bypass grafting, valvular surgery, or combined procedures: a systematic review with meta-analysis. Kardiol Pol 2018;76:10718 .

5. Abanoz M, Engin M. The effect of the relationship between post-cardiotomy neutrophil/lymphocyte ratio and platelet counts on early major adverse events after isolated coronary artery bypass grafting. Turk Gogus Kalp Dama 2021;29:36-44.

6. Eriş C, Sanrı US, Engin M, Yavuz Ş. Early postoperative results of on-pump coronary endarterectomy: is it still a controversy? Eur Res J 2021;7:248-55.

7. Buzby GP, Mullen JL, Matthews DC, Hobbs CL, Rosato EF. Prognostic nutritional index in gastrointestinal surgery. Am J Surg 1980;139:160-7.

8. Caputo F, Dadduzio V, Tovoli F, Bertolini G, Cabibbo G, Cerma K, et al. The role of PNI to predict survival in advanced hepatocellular carcinoma treated with Sorafenib. PLoS One 2020;15:e0232449.

9. Yang Y, Gao P, Song Y, Sun J, Chen X, Zhao J, et al. The prognostic nutritional index is a predictive indicator of prognosis and postoperative complications in gastric cancer: a meta-analysis. Eur J Surg Oncol 2016;42:1176-82.

10. Okada S, Shimada J, Kato D, Tsunezuka H, Teramukai S, Inoue M. Clinical significance of prognostic nutritional index after surgical treatment in lung cancer. Ann Thorac Surg 2017;104:296-302.

11. Onodera T, Goseki N, Kosaki G. Prognostic nutritional index in gastrointestinal surgery of malnourished cancer patients. Nihon Geka Gakkai Zasshi 1984;85:1001-5.

12. Engin M, Ozsin KK, Savran M, Guvenc O, Yavuz S, Ozyazicioglu AF. Visceral adiposity index and prognostic nutritional index in predicting atrial fibrillation after on-pump coronary artery bypass operations: a prospective study. Braz J Cardiovasc Surg. 2020 Dec 23. doi: 10.21470/1678-9741-2020-0044.

13. Sanrı U, Özsin K, Duman B, Toktaş F, Yavuz S . The relationship between prognostic nutritional index and carotid artery stenosis in patients with diagnosed carotid artery disease. CBUSBED. 2020;8:129-34.

14. O'Keefe S, Williams K, Legare JF. Hospital-Acquired Infections After Cardiac Surgery and Current Physician Practices: A Retrospective Cohort Study. J Clin Med Res. 2017;9:10-16.

15. Budczies J, Klauschen F, Sinn BV, Győrffy B, Schmitt WD, Darb-Esfahani S, et al. Cutoff finder: a comprehensive and straight forward Web application enabling rapid biomarker cutoff optimization. PLoS One 2012;7:e51862.

16. Chermesh I, Hajos J, Mashiach T, Bozhko M, Shani L, Nir RR, et al. Malnutrition in cardiac surgery: food for thought. Eur J Prev Cardiol 2014;21:475-83.

17. Sanchez JA, Sanchez LL, Dudrick SJ. Nutritional considerations in adult cardiothoracic surgical patients. Surg Clin North Am 2011;91:857-75.

18. Lomivorotov VV, Efremov SM, Boboshko VA, Nikolaev DA, Vedernikov PE, Lomivorotov VN, et al. Evaluation of nutritional screening tools for patients scheduled for cardiac surgery. Nutrition 2013;29:436-42.

19. Tepaske R, Velthuis H, Oudemans-van Straaten HM, Heisterkamp SH, van Deventer SJ, Ince C, et al. Effect of preoperative oral immune-enhancing nutritional supplement on patients at high risk of infection after cardiac surgery: a randomized placebo-controlled trial. Lancet 2001;358:696-701.

20. vanVenrooij LM, vanLeeuwen PA, Hopmans W, BorgmeijerHoelen MM, de Vos R, De Mol BA. Accuracy of quick and easy undernutrition screening tool short Nutritional Assessment Questionnaire, Malnutrition Universal Screening Tool, and modified Malnutrition Universal Screening Tool--in patients undergoing cardiac surgery. J Am Diet Assoc 2011;111:1924-30.

21. van Venrooij LM, de Vos R, Borgmeijer-Hoelen MM, Haaring $\mathrm{C}$, de Mol BA. Preoperative unintended weight loss and low body mass index in relation to complications and length of stay after cardiac surgery. Am J Clin Nutr 2008;87:1656-61.

22. Stoppe C, Goetzenich A, Whitman G, Ohkuma R, Brown T, Hatzakorzian R, et al. Role of nutrition support in adult cardiac surgery: a consensus statement from an International Multidisciplinary Expert Group on Nutrition in Cardiac Surgery. Crit Care 2017;21:131.

23. Bhamidipati CM, LaPar DJ, Mehta GS, Kern JA, Upchurch GR Jr, Kron IL, et al. Albumin is a better predictor of outcomes than body mass index following coronary artery bypass grafting. Surgery 2011;150:626-34.

24. Thourani VH, Keeling WB, Kilgo PD, Puskas JD, Lattouf $\mathrm{OM}$, Chen EP, et al. The impact of body mass index on morbidity and short and long term mortality in cardiac valvular surgery. $\mathrm{J}$ Thorac Cardiovasc Surg 2011;142:1052-61.

25. Bian C, Wu Y, Shi Y, Xu G, Wang J, Xiang M, et al. Predictive 
value of the relative lymphocyte count in coronary heart disease. Heart Vessels. 2010;25:469-73.

26. Korkmaz L, Kul S, Korkmaz AA, Akyüz AR, Ağaç MT, Erkan $\mathrm{H}$, et al. Increased leucocyte count could predict coronary artery calcification in patients free of clinically apparent cardiovascular disease. Turk Kardiyol Dern Ars 2012;40:223-8.

27. Sun XP, Li J, Zhu WW, Li DB, Chen H, Li HW, et al. Platelet to lymphocyte ratio predicts contrast-induced nephropathy in patients with ST-segment elevation myocardial infarction undergoing primary percutaneous coronary intervention. Angiology 2018;69:71-8.

28. Bagger JP, Zindrou D, Taylor KM. Leukocyte count: a risk factor for coronary artery bypass graft mortality. Am J Med.2003;115:660-3.

29. Wiedermann CJ, Wiedermann W, Joannidis M. Hypoalbuminemia and acute kidney injury: a meta-analysis of observational clinical studies. Intensive Care Med. 2010;36:1657-65.

30. de la Cruz KI, Bakaeen FG, Wang XL, Huh J, LeMaire SA, Coselli JS, et al. Hypoalbuminemia and long-term survival after coronary artery bypass: a propensity score analysis. Ann Thorac Surg 2011;91:671-5.
31. Nozoe T, Kimura Y, Ishida M, Saeki H, Korenaga D, Sugimachi K. Correlation of pre-operative nutritional condition with post-operative complications in surgical treatment for oesophageal carcinoma. Eur J Surg Oncol 2002;28:396-400.

32. Keskin M, Hayıroğlu MI, Keskin T, Kaya A, Tatlısu MA, Altay S, et al. A novel and useful predictive indicator of prognosis in ST-segment elevation myocardial infarction, the prognostic nutritional index. Nutr Metab Cardiovasc Dis 2017;27:438-46.

33. Lee SI, Ko KP, Choi CH, Park CH, Park KY, Son KH. Does the prognostic nutritional index have a predictive role in the outcomes of adult cardiac surgery? J Thorac Cardiovasc Surg 2020;160:145-53.e3.

34. Alexander KP, Newby LK, Cannon CP, Armstrong PW, Gibler WB, Rich MW, et al. American Heart Association Council on Clinical Cardiology; Society of Geriatric Cardiology. Acute coronary care in the elderly, part I: Non-ST-segment-elevation acute coronary syndromes: a scientific statement for health care professionals from the American Heart Association Council on Clinical Cardiology: in collaboration with the Society of Geriatric Cardiology. Circulation. 2007;115:2549-69. 